\title{
ACUTE ORAL IRON POISONING IN THE PEDIATRIC EMERGENCY DEPARTMENT
}

\author{
Ana Sofia Ferreira Lança ${ }^{1}$, Andreia Sofia Colaço Romana ${ }^{2}$, Ana Castelbranco Silva ${ }^{2}$, Maria Gomes Ferrera ${ }^{2}$. \\ ${ }^{1}$ Department of Pediatrics, Hospital de São Francisco Xavier, Centro Hospitalar de Lisboa Ocidental E.P.E., \\ Lisbon, Portugal; \\ ${ }^{2}$ Department of Pediatrics, Hospital Garcia de Orta, Almada, Portugal.
}

\begin{abstract}
Iron intoxication with oral supplements is a cause of morbidity and mortality in infancy. Detailed history must include timing and amount of elemental iron ingested. Peak serum iron determination may help clinical management and determine the prognosis.

A 14-year-old female adolescent with mood disorder and irregular followup was brought to the emergency department with incoercible vomiting and abdominal pain. She claimed to have ingested iron pills 12 hours before admission in a total amount of $27 \mathrm{mg} / \mathrm{kg}$ of elemental iron. She was hospitalized and intravenous fluids were started. Serum iron level at admission was $373 \mathrm{mcg} / \mathrm{dL}$. During hospitalization there was a resolution of symptoms, and she was discharged after a 24-hour observation period.

Intentional iron ingestions may convey a risk to suicidal intention and should be evaluated at an emergency department. Late arrival to the emergency department may difficult the clinical approach and worsen the prognosis.
\end{abstract}

\author{
ARTICLE HISTORY \\ Received 8 December 2021 \\ Accepted 14 January 2022
}

\section{KEYWORDS}

Iron, intoxication, adolescent.

\section{Introduction}

Iron intoxication is a potential cause of morbidity and mortality in children, because of toxic effects of elemental iron in different organs and systems. ${ }^{1,2}$ Most iron poisonings in pediatric age are due to unintentional ingestions, with no or minimal toxicity. On the other hand, intentional ingestions are associated with a higher mortality rate. ${ }^{2}$ Parents' false perception that iron supplements are innocuous may lead to careless storage making these products easily available to children and adolescents. ${ }^{3}$

The potency of iron preparations is related to the salt form used and the amount of elemental iron in each preparation. In a suspected iron poisoning the amount of elemental iron ingested influences the potential toxicity of it. Also, some preparations have a delayed-absorption rate, which also influences the clinical course.

Clinical manifestations of iron intoxication are classically described as a multiphasic syndrome in five stages, related to local and systemic toxicity and patient's clinical and laboratory features. . $^{1,4}$

The first stage is the gastrointestinal phase, which occurs within 6 hours of ingestion and is the result of direct injury to the gastrointestinal tract. Vomiting, diarrhea, abdominal pain, and gastrointestinal

Address for Correspondance: Ana Lança, Pediatrics Department, Hospital de São Francisco Xavier, Centro Hospitalar de Lisboa Ocidental E.P.E., Lisbon, Portugal

Email: anasofialanca@gmail.com

C2022 Pediatric Oncall hemorrhage may occur. Hypovolemic shock and metabolic acidosis with a positive anion gap may also develop during this stage and are a cause of death. Blood tests may reveal leukocytosis and hyperglycemia associated with hepatic injury secondary to iron absorption. ${ }^{5}$ The second stage is the latent phase, which usually occurs 6 to 24 hours after the ingestion. There is an apparent resolution of symptoms during this stage, presumably because of free circulating iron redistribution to the intracellular compartment. ${ }^{5}$ The third stage occurs within 6-72 hours after ingestion, and it is characterized by shock (distributive, hypovolemic or cardiogenic) and metabolic acidosis. Once iron reaches the intracellular compartment and causes cellular dysfunction, quelation therapy has little effect and the prognosis worsens. The fourth stage is distinguished by hepatotoxicity leading to liver failure and occurs 12 to 96 hours after ingestion. This is the second most common cause of death by iron intoxication. The final stage is associated with late sequela of gastrointestinal mucosa scarring causing bowel obstruction, which can occur within 2-8 weeks after ingestion. Vomiting is the most common manifestation secondary to gastric outlet obstruction. . $^{1,3,4,6}$

The initial management of a child with suspected iron intoxication includes gastric lavage with normal saline $^{5}$ if the patient presents within one hour after ingestion. ${ }^{5,7,8}$ Since activated charcoal is ineffective in iron absorption, this procedure is not recommended..$^{3,8}$ Peak serum iron concentration helps in estimating the severity of iron intoxication and guides the subsequent management. ${ }^{5}$ It should be measured four-to-six hours after ingestion, or eight hours after ingestion if slow- 
release iron supplements were taken. ${ }^{4}$ Values $<350$ $\mathrm{mcg} / \mathrm{dL}$ correlate with minimal toxicity and $>500 \mathrm{mcg} /$ $\mathrm{dL}$ with risk of serious systemic toxicity. ${ }^{6}$ Laboratory studies should also include complete blood count, coagulation studies, hepatic enzymes, blood urea nitrogen and creatinine, electrolytes and blood gases. 4,9 Abdominal radiograph may be useful in teenagers and children who ingested pills because iron tablets are radiopaque, therefore one might see and estimate the amount ingested. ${ }^{8}$

Deferoxamine is an iron chelator that binds free iron in the ferric state, which is responsible for iron toxicity, ${ }^{9}$ producing a water-soluble complex that is excreted by the kidneys. ${ }^{4}$ Indications to deferoxamine administration include severe symptoms, elevated anion gap metabolic acidosis and peak serum iron concentration superior to $500 \mathrm{mcg} / \mathrm{dL} .{ }^{4}$ The presence of deferoxamine-iron complexes in circulation may cause vin rose colored urine as a side effect. ${ }^{1}$

Patients that remain asymptomatic six hours after iron ingestion are unlikely to develop symptoms later on and may be discharged home. ${ }^{3}$

\section{Case Report}

A 14-year-old teenager girl was brought to the emergency department for incoercible vomiting and abdominal pain. She claimed to have ingested 13 ferrous sulfate pills, each containing $105 \mathrm{mg}$ of elemental iron per pill, 12 hours previously to the admission. This accounted for $27 \mathrm{mg} / \mathrm{kg}$ of elemental iron ingested. No stressor event was identified, and she denied suicidal intention at evaluation. The time of onset of the symptoms was not reported.

Previous history was relevant for irregular follow-up in pediatric psychiatry consultation for mood disorder and for an episode of acute alcohol intoxication motivating admission in the pediatric emergency department the year before.

On admission she was euthymic and hemodynamically stable. Physical examination revealed mild abdominal pain upon palpation and was otherwise unremarkable. Laboratory investigation showed leukocytosis (20.100/

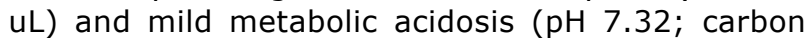
dioxide $42 \mathrm{mmHg}$; bicarbonate $21.6 \mathrm{mmol} / \mathrm{L}$; base excess $-4.5 \mathrm{mEq} / \mathrm{L}$; Anion Gap $15 \mathrm{mEq} / \mathrm{L}$; lactate $16 \mathrm{mg} / \mathrm{dL}$ ). Serum iron concentration was $373 \mathrm{mcg} /$ $\mathrm{dL} 12$ hours after ingestion. No tablets were seen on abdominal radiograph. The patient was hospitalized for surveillance and intravenous fluids. Presenting symptoms resolved and laboratory revaluation 24 hours after admission was normal. She was discharged home 36 hours after the ingestion.

\section{Discussion}

Here we present an intentional iron ingestion in a teenage girl. A previous study ${ }^{10}$ analyzed 113 patients admitted to the hospital with a discharge diagnosis of iron intoxication and concluded that 66 patients had intentional iron overdoses, most of them (80\%) females. This may be associated with the frequency of iron prescription in adolescent girls because of higher incidence of iron deficiency anemia in this group, making iron supplements more easily available. In the same study one-third of the patients revealed excess alcohol intake. Although our patient did not have alcohol intoxication at admission, the previous year she had had an alcohol intoxication that motivated admission in the pediatric emergency department.

As previously highlighted, the severity of the intoxication is intimately related to the amount of elemental iron ingested, so quantification at admission is of paramount importance. Halil et $\mathrm{al}^{6}$ performed a study to determine the minimum dosage of ingested elemental iron that could convey a risk of toxicity. The cut-off value that required medical management was $>28 \mathrm{mg} / \mathrm{kg}$. In our patient the presumable amount of elemental iron ingested was $27 \mathrm{mg} / \mathrm{kg}$ and the patient presented with gastrointestinal symptoms requiring hospital admission. Manoguerra et $\mathrm{al}^{3}$ recommended a higher threshold of presumable ingested iron of $40 \mathrm{mg} / \mathrm{kg}$ to require referral to a hospital unit if the patient only presents with mild symptoms. Besides, it is recommended that any patient with intentional ingestion in whom suicidal intention cannot be excluded should be evaluated at a hospital facility, ${ }^{9}$ similar to our case description.

Our patient presented with vomiting and abdominal pain, both described as the commonest presenting symptoms in a retrospective descriptive study performed by Singhi et $\mathrm{al}^{7}$ which reviewed patients brought to the emergency department with iron overdose. Eightythree percent of the patients presented with vomiting, $72 \%$ with diarrhea, and $10.7 \%$ with abdominal pain.

Serum iron levels peak at approximately 4-6 hours after ingestion. Dosing may allow inferring of iron presumable toxicity and aids in deciding whether more aggressive treatment will be needed. Our patient was admitted to the emergency department 12 hours after ingestion, and although serum iron level was determined, the value was not helpful in predicting the clinical outcome. Since ingestion was over an hour before admission, gastric lavage was not indicated. The patient was hospitalized for clinical surveillance and intravenous hydration was started because of the incoercible vomiting. She was discharged home 24 hours after admission since the symptoms did not evolve. A pediatric psychiatry consult was requested for follow-up.

As stated by Manoguerra et $\mathrm{al}^{3}$, in a suspected iron intoxication it is important to know the patient's intent, the dose and iron formulation ingested, the time of ingestion, the actual weight of the patient, whether any symptoms have developed and if there are any underlying medical conditions. This information will allow the clinician to evaluate and decide the appropriate therapeutic approach.

\section{Compliance with Ethical Standards}

Funding: None

Conflict of Interest: None

\section{References:}

1. Madiwale T, Liebelt E. Iron: not a benign therapeutic drug. Curr Opin Pediatr 2006; 18:174-179.

2. Lacouture PG, Wason S, Temple AR, et al. Emergency assessment of severity in iron overdose by clinical and laboratory methods. J Pediatr 1981;99(1)89-91.

3. Manoguerra AS, Erdman AR, Booze LL, Christianson G, et 
all. Iron Ingestion: an Evidence-Based Consensus Guideline for Out-of-Hospital Management. Clinical Toxicology 2005;43:6, 553-570

4. Liebelt EL. Acute iron poisoning. Uptodate 2019.

5. Baranwal AK, Singhi SC. Acute iron poisoning: management guidelines. Indian Pediatr. 2003 Jun;40(6):534-40. PMID: 12824662

6. Halil $\mathrm{H}$, Tuygun $\mathrm{N}$, Polat $\mathrm{E}$, et al. Minimum ingested iron cut-off triggering serious iron toxicity in children. Pediatr Int. 2019 May;61(5):444-448. doi: 10.1111/ped.13834. PMID: 30882955
7. Singhi SC, Baranwal AK, Jayashree M. Acute Iron Poisoning: Clinical Picture, Intensive Care Needs and Outcome. Indian Pediatr 2003;40(12):1177-82.

8. Aldridge MD. Acute iron poisoning: what every pediatric intensive care unit nurse should know. Dimens Crit Care Nurs. 2007 Mar-Apr;26(2):43-8; quiz 49-50. doi: 10.1097/00003465-200703000-00001. PMID: 17312404.

9. Anderson AC. Iron poisoning in children. Curr Opin Pediatr. 1994 Jun;6(3):289-94. doi: 10.1097/00008480199406000-00010. PMID: 7914796.

10. Kroeker S, Minuk GY. Intentional iron overdose: an institutional review. CMAJ. 1994;150(1):45-48. 